\title{
Depression in elderly persons subject to childhood maltreatment is not modulated by corpus callosum and hippocampal changes
}

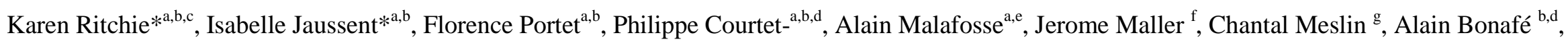

Emmanuelle Le Bars ${ }^{\text {b,d }}$, Nicolas Menjot de Champfleur ${ }^{\text {b,d }}$, Sylvaine Artero, ${ }^{\text {a,b }}$ and Marie-Laure Ancelin ${ }^{\mathrm{a}, \mathrm{b}}$.

*Authors K Ritchie and I Jaussent contributed equally to this article

${ }^{a}$ Inserm, U1061, Montpellier, F-34093, France

${ }^{\mathrm{b}}$ University of Montpellier 1, Montpellier, F-34000, France

${ }^{c}$ Faculty of Medicine, Imperial College, London, United Kingdom

${ }^{\mathrm{d}}$ CHRU Montpellier, Montpellier, France

e Department of Psychiatry, University of Geneva, CH-1211, Switzerland

${ }^{f}$ Monash Alfred Psychiatry Research Centre, The Alfred \& Monash University School of Psychology and Psychiatry, Melbourne, Australia

${ }^{\mathrm{g}}$ Centre for Mental Health Research, Australian National University, Canberra, Australia

\section{Corresponding Author}

Karen Ritchie

Inserm U1061, Neuropsychiatry: Epidemiological and Clinical Research,

La Colombière Hospital,

34093 Montpellier cedex 5, France

Tel: +33 4996145 68; Fax: +3349961 4579

Email: karen.ritchie@inserm.fr 
Key Words: corpus callosum, hippocampus, depression, magnetic resonance imaging, child abuse, elderly 


\section{ABSTRACT}

Childhood adversity has been observed to engender structural changes in the hippocampus and corpus callosum associated with increased risk for depression in childhood and early adulthood. This study investigated this association in the elderly. Corpus callosum area and hippocampal volume were measured from structural MRI in 427 community dwelling elderly. Information on childhood adversity was obtained in the course of a clinical examination using a questionnaire covering multiple aspects of abuse. Adjusted gender-stratified analyses found no significant reduction in corpus callosum area or hippocampal volume in exposed subjects. Previously observed associations between childhood adversity and late-life depression do not appear to be explained by structural brain changes.

\section{INTRODUCTION}

Clinical studies have consistently shown that childhood adversity is associated with increased vulnerability for depression in both childhood and early adulthood (Bernet and Stein 1999; Heim and Nemeroff 2001). Recently we have shown that this vulnerability persists into later life, even when multiple confounding effects including subsequent adverse life events have been taken into account (Ritchie et al. 2009). The neurobiological mechanism by which childhood stress heightens vulnerability to depression remains poorly understood, but appears to be the result of genetic predisposition as evidenced both by family and genotype interaction studies (Frodl et al. 2012; Gatt et al. 2009) interacting with adverse environmental exposures leading to alterations in brain development; notably by programming the glucocorticoid, noradrenergic and vasopressin stress response systems to over-react to new stressors (Shea et al.

2005; Teicher et al. 2005). These effects have been hypothesized to subsequently induce permanent structural changes which continue to compromise psychological adaptation, notably reduced white matter fibre connections and reduced development of the hippocampus (HC) and amygdala, corpus callosum (CC) and pre-frontal cortex (PFC) (Kaufman and Charney 2001). 
An early study of young children who had experienced maltreatment (De Bellis et al. 1999) reported smaller intracranial and cerebral volumes, larger lateral ventricular volume and smaller CC and HC compared to non-maltreated children. Subsequent studies have been unable to reproduce the finding of reduced HC volume in children (Carrion et al. 2001; Woon and Hedges 2008), however, reduced HC volume has generally been observed in young adults who have experienced maltreatment as children suggesting a delayed neurotoxic effect to chronic stress (Carrion et al. 2007). Three studies have examined amygdala volume with two showing no significant differences (Andersen et al. 2008; Bremner et al. 1997) and the third reduced volume only in females with dissociative identity disorder (Vermetten et al. 2006). Findings with regard to CC changes have been more clear-cut. Significant decreases in CC volume have been reported for children and adolescents by all studies (De Bellis and Kershaven 2003; Jackowski et al. 2008; Teicher et al. 2004) but one (Mehta et al.

2009). These changes have also been reported to persist into adulthood (Kitayama et al. 2007; Choi et al. 2009) and have been hypothesized to explain emotional regulation difficulties observed in adult victims (Choi et al. 2009). Smaller CC volumes are also found in babies born of alcoholic mothers

(Bookstein et al. 2007) which may constitute a confounding factor. Findings for the PFC have been inconsistent, with one reporting no difference (De Bellis et al. 1999) while two more recent studies found larger grey matter volume in the middle-inferior and ventral PFC regions (Richert et al. 2006; Carrion et al. 2009). Apart from differences in the adverse childhood events examined, variability in impact also appears to be due to "windows of vulnerability" in brain development, with the frontal cortex appearing to be maximally affected in late adolescence, the CC at 9-10 years and $\mathrm{HC}$ at 3-5 years (Andersen et al. 2008).

The aim of the present study is to ascertain from a large prospective epidemiological study whether elderly persons having experienced various types of childhood abuse and adversity still show brain changes similar to younger victims, and which may be postulated to exacerbate normal ageing related changes and vulnerability to mood disorders. The analysis focuses on structures most likely to have been affected in childhood and which current evidence suggests to persist into adulthood; the $\mathrm{CC}$ and $\mathrm{HC}$.

\section{METHODS AND MATERIALS}

\section{Subjects}


Community dwelling persons, 65 years and over, were recruited by random selection from the fifteen electoral roles of the Montpellier district between March 1999 and February 2001 as part of the ESPRIT study of late-life psychiatric disorder (Ritchie et al. 2004). Ethics approval for the study was given by the national ethics committee. All subjects gave their signed consent for participation in the study. Of the subjects initially drawn at random, $27.3 \%$ did not participate (of these $3.3 \%$ were excluded due to severe disability). Refusers were replaced by another subject drawn at random from the same electoral division such that each division is equally represented. Of the 472 persons having MRI CC and HC measures, and complete data on childhood adversity, 14 diagnosed with dementia and 31 left-handers were excluded leaving a sample of 427 subjects.

\section{Measures}

Participants were asked to attend a half-day examination by a neurologist and a project nurse at the Gui de Chauliac Neurology Hospital (Montpellier, France). The following procedures were carried out:

A standardized health interview covering present state of health, individual and family medical history, recent negative life events (occurring in the past year) and adult life-time experience of trauma. Depressive symptomatology was assessed using the Center for Epidemiologic Studies-Depression Scale (CES-D)

(Radloff 1977). Case-level late-life depression was defined as a score above the 16 cut-off point of the CES-D or current treatment with an anti-depressant at base-line. The Neale Adult Reading Test (NART) was included as an estimate of Intelligence Quotient (IQ) (Blair 1989).

Childhood environment. The Childhood Adversity Questionnaire (Ritchie et al. 2009) examining traumatic experiences during childhood was given to subjects for completion in the third wave of the study (four years after recruitment) by which time the study clinicians and project nurses had established close relationships with the participants, facilitating the request for sensitive information. Subjects were asked to respond yes or no to each item and in cases of doubt clinicians further probed responses. Items were grouped for this analysis into physical and/or sexual maltreatment; mental cruelty; mental disorder and substance abuse in parents; excessive sharing of parental problems; death of a parent; poverty; exposure to war or natural catastrophe. 
MRI imaging_A 1.5T GE Signa Imaging System (General Electric Medical Systems, Milwaukee, WI) was used to acquire a contiguous AC-PC aligned axial IR-prepared SPGR T1-weighted sequence for volumetric estimations $(\mathrm{TR}=12, \mathrm{TE}=2.8, \mathrm{IT}=600$, matrix size $=256 \times 256$, pixel spacing $=0.9375 \mathrm{x} 0.9375$ $\mathrm{mm}, \mathrm{NEX}=1$, slice thickness $=1.0 \mathrm{~mm})$. Slices were then converted to be isotropic $\left(0.9375 \mathrm{~mm}^{3}\right)$ and re-sliced to $1.00 \mathrm{~mm}^{3}$. CC outline was manually traced on the midline sagittal slice of the T1 images using anatomical landmarks in a hierarchical order and the Region of Interest (ROI) module of Analyze ${ }^{\mathrm{TM}} 9.0$ (Brain Imaging Resource, Mayo Clinic, MN, USA) on a Windows XP Professional workstation. The landmarks based on the midline sagittal slice were: i) no white matter or only minimal white matter in the cortical mantle surrounding the $\mathrm{CC}$; ii) the inter-thalamic adhesion; and iii) the transparent septum and the visibility of the aqueduct of Sylvius (30). Callosal areas were expressed in $\mathrm{mm}^{2}$.

Hippocampal ROIs were manually outlined on consecutive coronal slices and verified from axial and sagittal orientations (Maller et al. 2007). The anterior tip of the HC until the slice before the opening of the crus of the fornix (CF) was measured as the HC head and body and included the subiculum, CA1-(4) areas, and dentate gyrus (DG), as described by Watson et al. (Watson et al. 1997), and the HC tail was measured from the slice immediately posterior to that which represented the last slice according to the Watson protocol. The internal structure of the HC tail is the same as in the head and the body, whereby the cornu Ammonis has an analogous structure throughout, as does the gyrus dentatus. From coronal perspective, measuring the HC until the CF represents the part of the tail which coincides with the coronal section of the pulvinar (which is situated in a supero-medial position). Voluminous choroidal plexuses occupy portions of this region, hence care was taken to exclude them laterally from volumetric estimates. The HC was then followed posteriorly. On initial slices, the tail appears bulgy as an ovoid mass of gray matter on the infero-medial part of the lateral ventricle, and more posteriorly it lies flattened on the superior surface of the parahippocampal gyrus. The tail was outlined until the faciolar gyrus becomes the subsplenium gyrus curving around the postero-inferior margin of the splenium. The superior border was easy to differentiate from the crus of the fornix. The medial and inferior limits were also easily drawn because of the contrast between gray/white matter. Relevant images from standard atlases were referred to in order to ensure a consistent reference to the boundaries and relevant landmarks for these slices of $\mathrm{HC}$. All HC volumes are presented in $\mathrm{mm}^{3}$. 
Intra-rater and inter-rater reliability

CC and HC outlines were traced by two trained researchers blind to the study hypotheses, group assignment and subjects' identity. The reliability of the $\mathrm{CC}$ and $\mathrm{HC}$ measurements was assessed using a formula to calculate the intra- and inter-class correlations (intra-CC, intra $\mathrm{HC}$ and inter-CC, inter $\mathrm{HC}$ ) that presumes random selection of raters. The two researchers (JM, CM) each retraced five MRI images, which were randomly selected among the images previously traced, and five images which belonged to the group previously traced by the other researcher. Intra-CC was 0.957 for JM and 0.962 for CM. InterCC was 0.915. Intra-HC was 0.942 for JM and 0.970 for CM. Inter-HC was 0.939. All these values are well within acceptable limits.

Brain volume

Total brain volume (grey plus white matter) was computed for each subject using the 'segment' m-file of the SPM5 software (Wellcome Department of Cognitive Neurology, UK). All outputs were manually inspected to ensure accurate and valid data.

\section{Statistical analysis}

Midsaggital total CC area and total HC volume were expressed as ratios of total brain volume to control for differences in overall brain size. Using Shapiro-Wilk statistics, these two ratios were found to be normally distributed. The relationships between total CC area and HC volume and clinical and sociodemographic variables were tested using Pearson correlation coefficient for continuous variables and Student's t-test for categorical variables in order to identify potential confounders. Mean CC area and mean total HC volume ratios were examined in relation to childhood events by cross-sectional analysis of variance or covariance adjusted by age, gender and head injury. Analyses of covariance were performed to examine the effect of the interaction between two childhood events on mean CC area and total HC volume ratios. The reference group for individual events were persons with no adverse events. Logistic regression was used to estimate odds ratios (OR) and 95\% confidence intervals (CI) for the relationship between CC area and $\mathrm{HC}$ volume and depression 
relative to other known depression risk factors. Significance level was set at $\mathrm{p}<0.05$. Analyses were performed using SAS statistical software (version 9.2;

SAS Inc, Cary, North Carolina).

\section{RESULTS}

Table 1 shows the crude association between total CC area and HC volume and potential confounding and interactive factors according to previous research on child abuse. A significant negative association was found with age and gender for CC area and HC volume, whereas serious events occurring after childhood were associated with lower HC volume only. Life-time history of head injury was also found to be significantly associated with lower CC area only. No relationship was found between CC and HC volume and IQ, recent life events or parental alcohol abuse.

\section{Table 1}

Subsequent analyses are adjusted for age and life time head injury. Given gender differences previously observed in the relationship between CC area and $\mathrm{HC}$ volume and child abuse in younger populations, the analyses were stratified by sex (Table 2). No significant associations were found in women regardless of childhood event. In men physical or sexual abuse, mental disorder in parents, poverty and exposure to war or natural catastrophe were associated with higher $\mathrm{HC}$ volume.

\section{Table 2}

Logistic regression was used to examine to what extent $\mathrm{CC}$ area and $\mathrm{HC}$ volume may explain depression in late-life controlling for other known risk factors for depression (age, sex, and widowhood). While increased odds ratios for depression were observed in women (OR=2.34; 95\% CI 1.38-3.98; $\mathrm{p}=0.001)$, in persons experiencing recent adverse life-events $(\mathrm{OR}=1.9795 \%=[1.08-3.59], \mathrm{OR}=3.2095 \%=[1.73-5.91] \mathrm{p}=0.0007)$, for 1 and 2 recent traumatic 
events, respectively; and widows ( $\mathrm{OR}=1.85 ; 95 \%$ CI 0.93-3.70; $\mathrm{p}=0.081)$, no significant association was found for either CC area or HC volume ( $\mathrm{p}=0.82$ and 0.50 , respectively).

\section{DISCUSSION}

Both human and animal research have shown that both childhood abuse and childhood neglect are related to reduced CC area and HC volume in young adulthood (for review see McCrory et al. 2010), however little is known about the persistence of these structural changes in later life or their possible contribution to late-life psychopathology, notably depression. Our findings suggest that the structural differences observed in young adults who have experienced adverse childhood events are no longer present in the elderly, although we have previously established within the same cohort that these childhood events themselves do continue to constitute risk factors for late life depression (Ritchie et al. 2004). A recent study by Apfel et al. (Apfel et al 2011) found reduced HC volumes to be associated with current but not life-time posttraumatic stress disorder (PTSD) in Gulf War veterans (mean age 45 years, $85 \%$ men) suggesting that either a small hippocampus is a risk factor for failure to recover from trauma, or that effects on hippocampal volume are reversible after recovery. Our findings tend to support the latter hypothesis, given our observation that childhood events are associated with depression in later-life but not CC and HC decreases.

We observed, on the other hand, higher CC area and HC volume in men who had experienced poverty or mental disorder in parents as children, and higher HC volume in men who were exposed to war or natural disaster, or physical and sexual abuse. While compensation or resilience may be a tempting explanation for this, the actual increases in volume and area are extremely small and unlikely to be highly significant clinically. The central question in relation to our study is whether the association previously observed in this cohort between late-life psychopathology, notably mood disorder, and childhood adversity can be attributed to persistent reductions in CC and HC size. Our results suggest this is not the case; while our results confirmed previously 
established risk factors (female gender, widowhood, and adverse life events), CC area and hippocampal size do not contribute significantly to late-life depression although it is possible that there has been compensation from previous low levels which in turn in the past have given rise to functional changes. Dysfunction of stress regulation by the autonomic nervous system and the hypothalamus-pituitary-adrenal (HPA) axis are a possible example of this and constitute alternative pathways which should be explored in future studies as having possibly a more important role as vulnerability factors in late life psychiatric disorder.

To our knowledge this is the first study to have explored whether structural brain changes previously linked to adverse childhood events persist into late-life. The study's strength is the large well-phenotyped cohort which has permitted us to examine gender differences as well as a number of potential confounding variables including head injury and subsequent adverse events, the high quality of the structural measures and information on a wide range of childhood events. The principal weakness of the study is reliance on recall of childhood events; this being the basis, however, of all current knowledge of long-term effects in the absence of adequate birth cohort data. In our study we delayed enquiry about sensitive issues such as childhood abuse until the fourth wave of our study by which time the clinicians conducting the interviews had established positive relationships with participants. A questionnaire was used with a simple yes/no scoring system as this was found on pilot-testing to be less confronting than direct questioning. In cases of doubt about the event, the clinical staff further questioned subjects. Bias due to depressed subjects' ability to recall previous painful events is unlikely as positive associations were found with adult traumatic events. While birth cohort data would provide a more convincing case, such data is presently unavailable.

\section{Acknowledgements}

The ESPRIT Project is financed by the regional government of Languedoc-Roussillon, the Agence Nationale de la Recherche (ANR 07-LVIE-004) and an unconditional grant from Novartis. This study is also supported by France Alzheimer. 
None of the funding organization or sponsors played a role in the design and conduct of the study, in the collection, management, analysis, and interpretation of the data or in the preparation, review or approval of the manuscript.

\section{Financial disclosures. None reported}




\section{REFERENCES}

Andersen, S.L., Tomada, A., Vincow., A.S, Valente, E., Polcari, A., Teicher, M.H. 2008 Preliminary evidence for sensitive periods in the effect of childhood sexual abuse on regional brain development. J Neuropsych Clin Neurosci 20, 292-301.

Apfel, B.A., Ross, J., Hlavin, J., Meyerhoff, D.J, Metzler, T.J., Marmar C.R. 2011 Hippocampal volume differences in Gulf War veterans with current versus lifetime posttraumatic stress disorder symptoms. Biol Psychiatry 69, 541-548.

Bernet, C.Z., Stein, M.B. 1999 Relationship of childhood maltreatment to the onset and course of major depression in adulthood. Depress Anxiety 9,169-174.

Blair, JR, Spreen, O. Predicting premorbid IQ: a revision of the National Adult Reading Test. Clin Neuropsychol 1989; 3:129-136.

Bookstein, F.L, Connor, P.D., Huggins, J.E., Barr, H.M., Pimentel, K.D., Streissguth, A.P. 2007 Many infants prenatally exposed to high levels of alcohol show one particular anomaly of the corpus callosum. Alcohol Clin Exp Res 31, 868-879.

Bremner, J.D., Randall, P., Vermetten, E., Staib, L., Bronen, R.A., Mazure, C. 1997 Magnetic resonance imaging-based measurement of hippocampal volume in posttraumatic stress disorder related to childhood physical and sexual abuse - a preliminary report. Biol Psychiat 41, 23-32.

Carrion, V.G., Weems, C.F., Eliez, F., Patwardhan, A., Brown, W., Ray, R.D. 2001 Attenuation of frontal asymmetry in pediatric posttraumatic stress disorder. Biol Psychiatry 50, 943-951

Carrion, V.G., Weems, C.F., Reiss, A.L. 2007 Stress predicts brain changes in children: a pilot longitudinal study on youth stress, posttraumatic stress disorder and the hippocampus. Pediatrics 119, 509-516. 
Carrion, V.G., Weems, C.F., Watson, C., Eliez, S., Menon, V., Reiss, A.L. 2009 Converging evidence for abnormalities of the prefrontal cortex and evaluation of midsagittal structures in pediatric posttraumatic stress disorder: an MRI study. Psychiat Res 172, 226-234.

Choi, J., Jeong, B., Rohan, M.L., Polcari, A.M., Teicher, M.H. 2009 Preliminary evidence for white matter tract abnormalities in young adults exposed to parental verbal abuse. Biol Psychiatry 65, 227-234.

De Bellis, M.D., Baum, A.S., Birmaher, B., Keshaven, M.S., Eccard, C.H., Boring A.M. 1999 Developmental traumatology. Part I. Biological stress systems. Biol Psychiatry 45, 1259-1270

De Bellis, M.D., Keshaven, M.S. 2003 Sex differences in brain maturation in maltreatment-related pediatric posttraumatic stress disorder. Neurosci Behav Rev 27, 103-117.

Frodl T, Carballedo A, Fagan AJ, Lisiecka D, Ferguson Y, Meaney JF. 2012 Effects of early-life adversity on white matter diffusivity changes in patients at risk for major depression. J Psychiatry Neurosci 37,37-45

Gatt JM, Nemeroff CB, Dobson-Stone C, Paul RH, Bryant RA, Schofield PR, Gordon E, Kemp AH, Williams LM. 2009 Interactions between BDNF Val66Met polymorphism and early life stress predict brain and arousal pathways to syndromal depression and anxiety. Mol Psychiat 14,681-695

Heim, C., Nemeroff, C.B. 2001 The role of childhood trauma in the neurobiology of mood and anxiety disorders: preclinical and clinical studies. Biol Psychiatry. 49, 1023-39.

Jackowski, A.P., Douglas-Palumberi, H., Jackowski, M., Win, L., Schultz, R.T., Staib, L.W. 2008 Corpus callosum in maltreated children with posttraumatic stress disorder: a diffusion tensor imaging study. Psychiat Res 162, 256-261. 
Kaufman, J., Charney, D. 2001 Effects of early stress on brain structure and function: implications for understanding the relationship between child maltreatment and depression. Dev Psychopathol 13,451-71.

Kaufman J, Yang B-Z, Douglas-Palumberi H, Houshyar S, Lipschitz D, Krystal JH. 2004 Social supports and serotonin transporter gene moderate depression in maltreated children. Proc Natl Acad Sci 101, 17316-17321

Kitayama, N., Brummer, M., Hertz, L., Quinn, S., Kim, Y., Bremner, J.D. Morphologic alterations in the corpus callosum in abuse-related posttraumatic stress disorder. A preliminary study. 2007 J Nerv Ment Dis 195, 1027-1029.

Maller, J.J., Daskalaskis, Z.J., Fitzgerald, P.B. 2007 Hippocampal volumetrics in depression: the importance of the posterior tail. Hippocampus 17, 10231027.

McCrory, E., De Brito, S., Viding, E. 2010 Research review: the neurobiology and genetics of maltreatment and adversity. J Child Psychol Psychiat 51, 10791095.

Mehta, M.A., Golembo, N.I., Nosarti, C., Colvert, E., Mota, A., Williams, S.C.R. 2009 Amygdala, hippocampal and corpus callosum size following severe early institutional deprivation. J Child Psychol Psychiat 50, 943-951.

Radloff, L.S. 1977 The CES-D scale: a self-report depression scale for research in the general population. Appl Psychol Measurement 1, 385-401.

Richert, K.A., Carrion, V.G., Karchemskiy, A., Reiss, A.L. 2006 Regional differences of the prefrontal cortex in pediatric PTSD: an MRI study. Dep Anx 23, $17-25$.

Ritchie, K., Artero, S., Beluche, I., Ancelin, M.L., Mann, A., Dupuy, A.M., Boulenger, JP. 2004 Prevalence of DSM-IV psychiatric disorder in the French elderly population. Br J Psychiatry 184, 147-52. 
Ritchie, K., Jaussent, I., Stewar,t R., Dupuy, A-M., Courtet, P., Ancelin, M-L;, Mann,A., Dupuy, A-M., A., Malafosse, A., Boulenger, P. 2009 Association of adverse child environment and 5-HTTLPR genotype with late-life depression. J Clin Psychiatry 70, 1281-1288

Shea, A., Walsh, C., Macmillan, H., Steiner, M. 2005 Child maltreatment and HPA axis dysregulation: relationship to major depressive disorder and post traumatic stress disorder in females. Psychoneuroendocrinology. 30,162-178.

Teicher, M.H., Andersen, S.L., Polcari, A., Anderson, C.M., Navalta, C.P. 2002 Developmental neurobiology of childhood stress and trauma. Psychiatr Clin North Am 25, 397-426.

Teicher, M.H., Dumont, N.L., Ito, Y., Vaituzis, C., Giedd, J.N., Andersen, S.L. 2004 Childhood neglect is associated with reduced corpus callosum area. Biol Psychiat 56, 80-85

Vermetten, E., Schmal, C., Lindner, S., Loewenstein, R.J., Bremner, J.D. 2006 Hippocampal and amygdale volumes in dissociative identity disorder. Am J Psychiat 163, 630-636.

Watson, C., Jack., C.R., Jr., Cendes, F. 1997 Volumetric magnetic resonance imaging. Clinical applications and contributions to the understanding of temporal lobe epilepsy. Arch Neurol 54, 1521-1531.

Woon, F.L., Hedges, D.W. 2008 Hippocampal and amygdala volumes in children and adults with childhood maltreatment-related posttraumatic stress disorder: a meta-analysis. Hippocampus 18, 729-736. 

Ritchie. 17 